\title{
La contabilidad de costos como herramienta de gestión en la microempresa Yoha's Style
}

Cost accounting as a management tool in Yoha's Style microenterprise

Ruth Maryury Delgado Olaya. ${ }^{1}$, Mariana Marisol Yánez Sarmiento. ${ }^{2}$, Betzy Lizbeth Orellana Arévalo. ${ }^{3}$, Jessenia Maribel Camacho Yunga. ${ }^{4} \&$ Kenia Elizabeth Espinoza Criollo. ${ }^{5}$

\begin{abstract}
DOI: https://doi.org/10.33262/concienciadigital.v4i4.2.1940

Introduction. The growth of micro-enterprises is conditioned by the management that is applied. These should not be tied to the tax category or its size to implement management tools such as accounting, which provides information for decision-making. Objective. Analyze cost accounting as a management tool in the Yoha's Style microenterprise, using economic and financial analysis techniques to determine its profitability during the January 2020 period. Methodology. The study has a non-experimental quantitative approach since the data is not manipulated, it is considered a cross-sectional, descriptive study. There was information on real and nominal accounts provided by the owner and accountant of the microenterprise. The results obtained in the survey of 416 microentrepreneurs in the province of El Oro were taken as a basis. Information

\footnotetext{
1 Universidad Metropolitana, Carrera de Contabilidad y Auditoría, El Oro - Ecuador, https://orcid.org/0000-0001-7159-3729, rdelgado@umet.edu.ec

2 Universidad Metropolitana, Carrera de Contabilidad y Auditoría, El Oro - Ecuador, https://orcid.org/0000-0002-1284-1478, myanez@umet.edu.ec

3 Universidad Metropolitana, Carrera de Contabilidad y Auditoría, El Oro - Ecuador, https://orcid.org/0000-0002-9050-1989, betzy.orellana@est.umet.edu.ec

4 Universidad Metropolitana, Carrera de Contabilidad y Auditoría, El Oro - Ecuador, https://orcid.org/0000-0003-0197-2074, jessenia.camacho@est.umet.edu.ec

5 Universidad Metropolitana, Carrera de Contabilidad y Auditoría, El Oro - Ecuador, https://orcid.org/0000-0002-1229-7206, kenia.espinoza@est.umet.edu.ec
} 
processing was carried out using the VISUALFAC accounting system and the Microsoft Excel program until obtaining financial information and subsequent quantitative analysis. Results. Among the results, it is evident that the operational performance of the microenterprise in relation to the equity is very low, obtaining only $4 \%$ as a return to the owners; Likewise, in the net profitability of the asset and financial profitability it generates only 3\% and 4\% respectively. Conclusions. Cost accounting provides quantitative information to managers on the resources, obligations and assets that the microentrepreneur owns.

Keywords: costs, management, profitability, microenterprise.

\section{Resumen}

Introducción. El crecimiento de las microempresas se ve condicionada por la gestión que se aplique. Estas no deben estar atadas a la categoría tributa o su tamaño para implementar herramientas de gestión como la contabilidad, que suministra información para la toma de decisiones. Objetivo. Analizar la contabilidad de costos como herramienta de gestión en la microempresa Yoha's Style utilizado técnicas de análisis económico y financiero, para la determinación de su rentabilidad durante el período de enero 2020. Metodología. El estudio tiene un enfoque cuantitativo de tipo no experimental ya que no se manipulan los datos, se considera un estudio de corte transversal, descriptivo. Se contó con información de cuentas reales y nominales proporcionada por el propietario y contador de la microempresa. Se tomó como base los resultados obtenidos en la encuesta realizada a 416 microempresarios en la provincia de El Oro. El procesamiento de la información se realizó utilizando el sistema contable VISUALFAC y el programa Microsoft Excel hasta obtener la información financiera y posterior análisis cuantitativo. Resultados. Entre los resultados se evidencia que el rendimiento operacional de la microempresa con relación al patrimonio es muy bajo al obtener tan sólo el $4 \%$ como retorno a los propietarios; asimismo, en la rentabilidad neta del activo y rentabilidad financiera genera tan sólo el $3 \%$ y $4 \%$ respectivamente. Conclusiones. La contabilidad de costos facilita información cuantitativa a los administradores sobre los recursos, obligaciones y patrimonio que el microempresario posee.

Palabras claves: costos, gestión, rentabilidad, microempresa.

\section{Introducción}

Las microempresas son y han sido parte del sector productivo y económico de los países desarrollados y en miras de desarrollo. Algunos entendidos en materia económica han categorizado a estos negocios como la máxima expresión en un proceso de recuperación y reordenamiento en la economía en los países. Las empresas en América Latina corresponden al $99.5 \%$ entre las Mipymes, de las cuales el $88.4 \%$ son microempresas; dicha situación se contrapone con el 25\% de participación que generan en la contribución del PIB en la región (Correa et al., 2018); su principal finalidad consiste en crear empleo 
a personas que no han podido insertarse en el ámbito laboral (García, 2021). En el caso de Ecuador; desde hace una década, las microempresas según su estructura representan entre el $89 \%$ y $91 \%$ del total de empresas, liderando entre las pequeñas, medianas y grandes empresas (Instituto Nacional de Estadísticas y Censos [INEC], 2021). Las microempresas se constituyen en la principal opción en la población para mitigar el desempleo y generar ingresos.

La mayoría, de microempresarios inician sus actividades de forma empírica, ya que desconocen las funciones de administración, contabilidad y finanzas, indispensables para la consecución de objetivos y metas (Gutiérrez \& Gutiérrez, 2017). En este mismo contexto, Santana (2017) manifiesta que la mortalidad de las microempresas se encuentra asociada a algunos factores, como la falta de gestión, falta de competitividad; entre otros; sin embargo, considera que una de las principales razones para el cierre de un pequeño negocio es la quiebra financiera. En este sentido, el crecimiento y desarrollo microempresarial se ven amenazados; y consecuentemente, la producción y desempeño económico a nivel nacional. Sumando a lo anterior para Texis et al. (2016) las microempresas son un sector que se enfrentan a diversas restricciones como la falta de planeación, formación empresarial, seguridad social y acceso a financiamiento. Todos estos antecedentes dificultan aún más la supervivencia de este sector que tiende a desaparecer al corto plazo.

El sector de manufactura es considerado como una de las actividades primordiales en la contribución al crecimiento en la economía en el Ecuador (Yasuní, 2016); pues contribuyó en el año 2016 con el 12,5\% al PIB y con un 14,78\% a la generación de empleo de la concentración de mano de obra nacional, empleando hasta 100 mil trabajadores en el año 2014. En el Ecuador existen 870 establecimientos que se dedican a la producción de calzado, para potenciar estos pequeños negocios en el corto tiempo, es imperioso aplicar estrategias de crecimiento, creación de información, generación de valor y reinversión de rentas (Morales et al., 2017). En la provincia de El Oro los ingresos anuales, bordearon los $\$ 139$ millones debido al aumento de la manufacturación (Yasuní, 2016). En tal sentido, se toma como objeto de estudio la microempresa "Yoha's Style" dedicada a la fabricación de calzado en la ciudad de Machala, dada la importancia que tiene el sector de la producción en la provincia y el país.

La microempresa "Yoha's Style" fabrica calzado de dama, cuenta con seis procesos: recepción de materia prima, cortada, entaconado, pegado, secado, limpieza y empaquetado. En términos tributarios esta categorizada como microempresa no obligada a llevar contabilidad, condición que la ha atado a la idea de no aplicar el proceso contable ni el posterior análisis de sus estados financieros. Al igual que la microempresa objeto de estudio, en la provincia de El Oro, de acuerdo con los resultados obtenidos en la encuesta realizada a 416 microempresas el $60 \%$ mencionan que no llevan un control de sus operaciones diarias de ventas, gastos, adquisición de activos; el 36\% responden que utiliza un cuadernillo para el control de sus ventas y gastos; y el 11\% manifiesta que no lleva ningún registro de sus operaciones. 
El presente estudio considera elementos imprescindibles para la toma de decisiones, como la contabilidad de costos y el análisis financiero, métodos para conocer la eficiencia económica, recursos utilizados en la producción, la productividad del trabajo realizado y crecimiento empresarial. En tal virtud el objetivo de esta investigación consiste en analizar la contabilidad de costos como herramienta de gestión en la microempresa "Yoha's Style" utilizado técnicas de análisis económico y financiero para la determinación de su rentabilidad durante enero 2020. La investigación está direccionada a fortalecer los conocimientos de los lectores que estén interesados en el uso y aplicación de la contabilidad de costos como herramienta de gestión en las microempresas, ya que hoy en día existen un sin número de microempresarios que aún desconocen la importancia de la información en los estados financieros y los beneficios que se obtienen con su aplicación.

\section{Resultados y Discusión}

La contabilidad es una herramienta de gestión fundamental para la toma de decisiones y el control de los procesos; ya que, a través de ella se pueden verificar las variables que afectan el desempeño de los administradores (Medina et al., 2018); cuantificar los costos de producción, y/o servicios, calcular el precio de mercado y el máximo valor de satisfacción de los usuarios (Escobar et al., 2021). Actualmente las empresas requieren producir al menor costo y ofrecer al mejor precio de competencia sus productos para mantenerse en el mercado a largo plazo. Especialmente el sector de la producción necesita la información sobre la inversión realizada, proporcionar datos que contribuyan a futuras proyecciones de inversión, elaborar informes para la toma de decisiones, valoración de los inventarios, control de costos, la medición de los ingresos-costos-beneficios y analizar la rentabilidad de los productos.

En este sentido; la contabilidad de costos permite a la administración en las empresas planificar, resumir, categorizar, acumular y controlar los costos asociados a la inversión en la producción y obtener datos reales y oportunos sobre la fabricación, comercialización, gestión y financiamiento de las operaciones productiva. Es en las empresas industriales, donde esto se considera clave (Mera Morocho, 2018 citado en Hurtado, 2020), para un mejor funcionamiento de sus procesos productivos, la alta gerencia necesita de la información que esta les brinda para controlar sus costos, proyectar presupuestos, tomar decisiones que influyen en la economía de la empresa y relacionar la inversión que debe ser recuperada.

En la contabilidad de costos se observan algunos métodos de aplicación que conllevan al control de los costos invertidos en la producción de un producto; para ello se presentan tres tipos de costos: a) materiales o materia prima directa, conocido como el principal elemento en la producción ; b) mano de obra directa, que se relaciona con el esfuerzo físico o intelectual del ser humano; y, c) los costos indirectos de fabricación, que se atribuyen a los materiales y mano de obra indirectos, no relacionados directamente en el proceso de fabricación y los complementos que necesita el producto para terminar su fabricación. Los elementos del costo se tratan en diferentes sistemas como por ejemplo 
los tradicionales: por órdenes de producción y por procesos. El primero según Quezada et al., (2020) parte de un conjunto de pedidos que esperan ser producidos con la ayuda de máquinas o en forma manual de manera cronológica, reduciendo tiempos de entrega y procesos al menor costos posible. Por el contrario; en el segundo según Villarreal y Rincón (2009) se inicia con un estudio de mercado donde se acumulan los costos incurridos en cada departamento, sección o etapas, al término de la fabricación se suman todos los costos hasta obtener el producto terminado.

El sistema de costos utilizado tiene influencia directa en el desempeño de las actividades en las empresas; por lo tanto, su estructura debe estar orientado a documentar la salud financiera, la gestión de la cadena de valor en relación a los recursos invertidos, dependiendo de la necesidad de cada empresa y de alcanzar mayor eficiencia empresarial a nivel estratégico, táctico y operativo (Mejía \& Higuita, 2015). De la misma forma para Kato (2013) un sistema contable refleja la realidad económica, financiera y suministra información suficiente a los usuarios potenciales. En el estudio de Porporato (2015) afirma que a través de los sistemas de contabilidad de gestión la información que proveen reduce la incertidumbre, entendiéndose como el riesgo; al obtener información requerida e información disponible en las empresas. Así mismo Baena (2014) sostiene que la información de los estados financieros implica los resultados de la gestión y desempeño de administradores en el tiempo. Estos resultados deben ser medidos de acuerdo con la industria a la cual pertenecen; sin embargo, un análisis financiero para que sea fiable, deberá tener el conjunto completo de estados financieros.

Con lo que coincide Vega y Tello (2013) "Cada año las empresas presentan sus estados financieros con el fin de comunicar los resultados obtenidos durante ese periodo para facilitar la toma de decisiones de los diferentes usuarios de la información contable"(p. 263). En el caso de las microempresas no obligadas a llevar contabilidad, sólo respaldan sus operaciones en anotaciones de ingresos y gastos, dicha situación limita el proceso de gestión acertada en la toma de decisiones. Una gestión eficiente en las empresas se define a través de la base contable que es indispensable para el análisis financiero como resultado de la eficiencia y trabajo empresarial. En el mismo contexto Nava y Marbelis (2009) mencionan que los objetivos del análisis financiero se fundamentan en la medición del nivel de solvencia, liquidez, rentabilidad y, en general la situación financiera actual de la empresa; es decir, el cálculo de indicadores o razones financieras, que permiten un diagnóstico e interpretación de los resultados.

La relevancia del análisis financiero permite conocer los aspectos económicos y financieros en las empresas; en qué circunstancias operan en relación a aspectos de liquidez, endeudamiento, solvencia, eficiencia, rentabilidad y rendimiento de acuerdo a la actividad empresarial (Nava \& Marbelis, 2009). A continuación, se contextualizan los indicadores financieros que son utilizados en la presente investigación. En la tabla 1 se evidencian los indicadores de liquidez, que se aplican para determinar los activos que se convierten en efectivo fácilmente e inmediatamente (Muñoz, 2018); es decir, miden la capacidad que poseen las empresas para pagar sus deudas a corto plazo (Superintendencia de Compañías del Ecuador, 2021). 
Tabla 1

Indicador de Liquidez

\begin{tabular}{lr}
\hline \multicolumn{1}{c}{ Indicadores } & Fórmulas \\
\hline 1. Liquidez corriente: & Activo Corriente / Pasivo Corriente \\
2. Prueba ácida: & Activo Corriente - Inventario / Pasivo Corriente \\
\hline Fuente: Superintendencia de Compañías del Ecuador (2021)
\end{tabular}

Los indicadores de solvencia hacen referencia al endeudamiento y apalancamiento de las empresas. En la tabla 2 se especifican los indicadores de solvencia (Wild et al., 2007), que reflejan la capacidad de pago de las empresas a largo plazo; además, muestra la participación de los acreedores y terceros en el financiamiento asumido por la entidad empresarial.

Tabla 2

Indicador de Solvencia

\begin{tabular}{ll}
\hline \multicolumn{1}{c}{ Indicadores } & \multicolumn{1}{c}{ Fórmula } \\
\hline 1. Endeudamiento del Activo: & Pasivo Total / Activo Total \\
2. Endeudamiento Patrimonial: & Pasivo Total / Patrimonio \\
3. Endeudamiento del Activo Fijo: & Patrimonio / Activo Fijo Neto \\
4. Apalancamiento: & Activo Total / Patrimonio \\
5. Apalancamiento Financiero: & (UAI / Patrimonio) / (UAII / Activos Totales) \\
\hline
\end{tabular}

Fuente: Superintendencia de Compañías del Ecuador (2021)

Por el contrario, en la tabla 3 se mencionan los indicadores de rentabilidad (Barreto, 2020), que reflejan el resultado del capital invertido por los accionistas o propietarios a través del dinero que mantienen en la empresa, cuyos valores se evidencian en las ganancias o pérdidas. Asimismo estos indicadores permiten evaluar la capacidad de la administración en controlar los costos y gastos (Superintendencia de Compañías del Ecuador, 2021).

Tabla 3

Indicador de Rentabilidad

\begin{tabular}{ll}
\hline \multicolumn{1}{c}{ Indicadores } & \multicolumn{1}{c}{ Fórmula } \\
\hline 1. Rentabilidad Neta del Activo: & (Utilidad Neta / Ventas) *(Ventas / Activo Total) \\
2. Margen Bruto: & Ventas Netas - Costo de Ventas / Ventas \\
3. Margen Operacional: & Utilidad Operacional / Ventas \\
4. Rentabilidad Neta de Ventas: & Utilidad Neta / Ventas \\
5. Rentabilidad Operacional del & (Utilidad Operacional / Patrimonio) \\
Patrimonio: & (Ventas / Activo) * (UAII / Ventas) * (Activo / \\
6. Rentabilidad Financiera: & Patrimonio) *(UAI / UAII)*(UN / UAI) \\
\hline
\end{tabular}

Fuente: Superintendencia de Compañías del Ecuador (2021) 
En el presente estudio el tipo de investigación tiene un enfoque cuantitativo, pues como menciona Hernández et al. (2014) representa un proceso recolector y analítico de datos con pocos márgenes de error, la producción de datos estadísticos controla la generación de respuestas y resultados positivos. Es de tipo no experimental ya que no se manipulan los datos. Se considera un estudio de corte transversa, descriptivo. Los resultados obtenidos proporcionan información a la empresa Yoha's Style, así como aquellas empresas que poseen las mismas características, como referencia de la información que otorga la contabilidad de costo y posterior análisis financiero. Esta investigación toma como base los resultados obtenidos en la encuesta realizada a 416 microempresarios en la provincia de El Oro, vinculadas en el proyecto de investigación "Herramientas Financieras" de la carrera Contabilidad y Auditoría de la Universidad Metropolitana, sede Machala, cuyos datos motivó a tomar como muestra a la microempresa Yoha's Style para sugerir la aplicación del proceso contable de acuerdo con sus necesidades. En el proceso investigativo se utilizó fuentes primarias a través de una entrevista a la propietaria y contador de la microempresa Yoha's Style. En los datos obtenidos está el respectivo inventario inicial de los bienes, valores, derechos y obligaciones, para iniciar el proceso contable. Para el procesamiento de los datos se utiliza el sistema contable VISUALFAC y el análisis e interpretación de los resultados por medio del programa Microsoft Excel 2019 (v19.0).

En la tabla 4 se observan los elementos que intervienen en el proceso operativo de producción de la microempresa, muestra los resultados de la agrupación de las cuentas en el estado de costos de producción y ventas. En este estado se presentan los costos de la producción; es decir, la inversión que realizan los propietarios con relación a las compras de materia prima, mano de obra y costos indirectos de fabricación. Para Kato Maldonado (2013) el análisis del ciclo de capital industrial permite explicar, cómo la producción de mercancías es producto del trabajo, el desgaste físico de la maquinaria y la velocidad con que son transformadas las materias primas, en este sentido, el proceso de fabricación es determinante en los costos totales de los productos terminados.

Tabla 4

Estado de Costos de Producción y Ventas (expresado en \$)

\begin{tabular}{|c|c|c|c|c|c|}
\hline \multicolumn{6}{|c|}{ MICROEMPRESA YOHA'S STYLE } \\
\hline \multicolumn{6}{|c|}{ DEL 01 AL 31 DE ENERO DEL 2020} \\
\hline 1.01.03.06 & & Materiales directos utilizados & & $\$$ & 716,50 \\
\hline 1.01.03.06.03 & & Inventario inicial de materia prima directa & $\$ 19.036,16$ & & \\
\hline 1.01.03.06.02 & $(+)$ & Compra de materia prima directa & 716,50 & & \\
\hline 1.01.03.06.05 & $(=)$ & Materiales disponibles para la producción & $\$ 19.752,66$ & & \\
\hline 1.01.03.06.06 & $(-)$ & Inventario final de materia prima directa & $\$ 19.036,16$ & & \\
\hline \multirow[t]{2}{*}{5.2 .02 .01 .01} & $(+)$ & Mano de obra directa & & $\$$ & 249,67 \\
\hline & $(=)$ & Costo primo & & $\$$ & 966,17 \\
\hline 5.1 .01 .02 .01 & $(+)$ & Costos indirectos de fabricación & & $\$$ & 234,85 \\
\hline
\end{tabular}


Tabla 4

Estado de Costos de Producción y Ventas (expresado en \$) (continuación)

\begin{tabular}{|c|c|c|c|c|c|c|}
\hline \multicolumn{7}{|c|}{ MICROEMPRESA YOHA'S STYLE } \\
\hline \multicolumn{7}{|c|}{ DEL 01 AL 31 DE ENERO DEL 2020} \\
\hline 1.01.03.06.04 & & Materia prima indirecta utilizada & $\$$ & 116,53 & & \\
\hline 1.01.03.06.05 & $(+)$ & INV. Inicial de materia prima indirecta & $\$$ & $3.285,90$ & & \\
\hline 1.01.03.06.02 & $(+)$ & Compras de materias primas indirectas & $\$$ & - & & \\
\hline 1.01.03.06.05.06 & $(=)$ & Materiales indirectos disponibles & $\$$ & $3.285,90$ & & \\
\hline 1.01.03.06.05.07 & $(-)$ & INV. Final de materia prima indirecta & $\$$ & $3.169,37$ & & \\
\hline 5.2.02.01.01.01 & $(+)$ & Mano de obra indirecta & & & $\$$ & 139,50 \\
\hline 5.1.01.02.01.01 & $(+)$ & Otros costos indirectos de fabricación & & & $\$$ & 29,57 \\
\hline 5.2 .02 .06 .01 & & Servicios básicos & $\$$ & 18,56 & & \\
\hline 1.02.01.12.01 & & Depreciación & $\$$ & 11,01 & & \\
\hline 5.1.01.02.01.02 & $(=)$ & Costos de fabricación del período & & & $\$$ & $1.251,76$ \\
\hline 1.01.03.06.06 & $(+)$ & INV. Inicial de productos en proceso & & & $\$$ & - \\
\hline 5.1 .01 .02 .01 & $(=)$ & Costo de producción en proceso disponible & & & $\$$ & $1.251,76$ \\
\hline 1.01.03.06.06 & $(-)$ & Inventario final de productos en proceso & & & $\$$ & - \\
\hline 1.01.03.06.05 & $(=)$ & Costo de producción de productos terminados & & & $\$$ & $1.251,76$ \\
\hline 1.01.03.06.05 & $(+)$ & INV. Inicial de productos terminados & & & & $31.500,00$ \\
\hline 1.01.03.06.05.01 & $(=)$ & Productos terminados disponibles para la venta & & & & $32.751,76$ \\
\hline \multirow[t]{2}{*}{ 1.01.03.06.05.01.01 } & $(-)$ & INV. Final de productos terminados & & & & $20.220,00$ \\
\hline & & Costo de producción y ventas & & & & $12.531,76$ \\
\hline
\end{tabular}

Fuente: Microempresa Yoha's Style, enero 2020

La tabla 5, muestra la estructura financiera y el análisis vertical de la microempresa; los recursos con los que cuenta la microempresa ascienden a $\$ 106.739,35$; mientras que el financiamiento con terceros es del $\$ 19.044,12$ y un capital propio de $\$ 87.695,23$. Los activos corrientes representan el 66,89\% del valor total de recursos; por el contrario, el activo no corriente el 33,11\% esto con relación al total de activos. Asimismo, se evidencia que la cuenta más representativa de los activos corrientes es la de inventarios con el 42,92\%; es decir, la microempresa Yoha's Style tiene su mayor inversión en el inventario de materia prima; esta situación tiene implicaciones como lo manifiesta Laveriano (2010) que en estos casos se producen costos altos en el almacenamiento, aumentan las mermas y los inventarios perecederos, lo que conlleva a una menor calidad de los productos que se ofrecen.

Dentro del grupo de activo no corriente; la cuenta de edificios es de mayor relevancia, ya que el $18,74 \%$ representa el total de los activos; es decir, la empresa posee instalaciones para el funcionamiento y desempeño de las actividades de la microempresa. Este rubro comprende para Palacios y Barreto (2014) el conjunto de los bienes que posea el ente económico, con la intención de utilizarlos permanentemente en el giro ordinario de sus negocios o que apoyan la gestión en la producción de bienes. Con respecto a los pasivos 
corrientes representan el 3,79\% en cuentas y documentos por pagar, obligaciones de impuestos, provisiones por pagar, obligaciones con el IESS y participación a trabajadores; por el contrario, los pasivos no corrientes el 14,05\%; la microempresa Yoha's Style mantiene mayor deuda a largo plazo. Situación óptima para la microempresa; ya que, según los autores Genoni y Zurita (2004) una empresa que decidiera financiar sus operaciones con deuda de corto plazo, no tendría capital de trabajo. En el último grupo del balance general corresponde al patrimonio en la cuenta capital el 79,16\%; y, el 3\% en ganancias, respectivamente con respecto al análisis del grupo.

Tabla 5

Estado de situación financiera (expresado en \$)

\begin{tabular}{|c|c|c|c|}
\hline \multicolumn{4}{|c|}{ MICROEMPRESA YOHA'S STYLE } \\
\hline \multicolumn{4}{|c|}{ AL 31 DE ENERO DEL 2020} \\
\hline & & & Vertical \\
\hline & & & VS Activo \\
\hline 1. & ACTIVO & & \\
\hline 1.01 . & ACTIVO CORRIENTE & & \\
\hline 1.01 .01 & Efectivo y equivalente al efectivo & $\$ 25.344,70$ & $23,74 \%$ \\
\hline 1.01.02.06.01 & Cuentas por cobrar clientes & $\$ 240,00$ & $0,22 \%$ \\
\hline 1.01.03.06.03 & Inventario de materia prima directa & $\$ 22.420,91$ & $21,01 \%$ \\
\hline 1.01.03.06.04 & Inventario de materia prima indirecta & $\$ 3.169,37$ & $2,97 \%$ \\
\hline \multirow[t]{2}{*}{ 1.01.03.06.05 } & Inventario de producto terminado & $\$ 20.220,00$ & $18,94 \%$ \\
\hline & Total activos corrientes & $\$ 71.394,98$ & $66,89 \%$ \\
\hline 1.02 & ACTIVO NO CORRIENTE & & \\
\hline 1.02 .01 & Propiedad, planta y equipo & & \\
\hline 1.02.01.02. & Edificios & $\$ 20.000,00$ & $18,74 \%$ \\
\hline 1.02.01.05. & Muebles y enseres & $\$ 1.580,00$ & $1,48 \%$ \\
\hline 1.02.01.06. & Maquinaria y equipo & $\$ 2.250,00$ & $2,11 \%$ \\
\hline 1.02.01.08. & Equipos de computación & $\$ 1.200,00$ & $1,12 \%$ \\
\hline & Vehículos, equipo de transporte y equipo & & \\
\hline 1.02.01.09. & camionero & $\$ 10.500,00$ & $9,84 \%$ \\
\hline \multirow[t]{3}{*}{ 1.02.01.12.01 } & (-) Depreciación acumulada de maquinaria & $-\$ 185,63$ & $-0,17 \%$ \\
\hline & Total activos no corrientes & $\$ 35.344,37$ & $33,11 \%$ \\
\hline & Total general del activo & $\$ 106.739,35$ & $100,00 \%$ \\
\hline 2. & PASIVOS & & \\
\hline 2.01 & PASIVOS CORRIENTES & & \\
\hline 2.01 .03 & Cuentas y documentos por pagar & $\$ 400,00$ & $0,37 \%$ \\
\hline 2.01.07.02.02 & Impuesto por pagar IVA & $\$ 1.901,32$ & $1,78 \%$ \\
\hline 2.01.07.03 & Obligaciones con el IESS & $\$ 274,06$ & $0,26 \%$ \\
\hline 2.01.07.04 & Provisiones por pagar & $\$ 903,48$ & $0,85 \%$ \\
\hline \multirow[t]{2}{*}{2.01 .07 .05} & Participación a trabajadores & $\$ 565,26$ & $0,53 \%$ \\
\hline & Total pasivos corrientes & $\$ 4.044,12$ & $3,79 \%$ \\
\hline
\end{tabular}


Tabla 5

Estado de situación financiera (expresado en \$) (continuación)

\begin{tabular}{llrr}
\hline \multicolumn{3}{c}{ MICROEMPRESA YOHA'S STYLE } \\
\hline \multicolumn{1}{c}{ AL 31 DE ENERO DEL 2020 } \\
2.02. & PASIVO NO CORRIENTE & \\
& Obligaciones con instituciones financieras - & \\
2.02 .01 & préstamo & $\$ 15.000,00$ & $14,05 \%$ \\
& Total pasivos no corrientes & $\$ 15.000,00$ & $14,05 \%$ \\
3.01 .01$. & Total de pasivos & $\$ 19.044,12$ & $17,84 \%$ \\
3.07 .01 & PATRIMONIO & & \\
& Capital & $\$ 84.492,06$ & $79,16 \%$ \\
& Ganancia neta del período & $\$ 3.203,17$ & $3,00 \%$ \\
& Total patrimonio & $\$ 87.695,23$ & $82,16 \%$ \\
& Pasivo + patrimonio & $\$ 106.739,35$ & $100,00 \%$ \\
\hline
\end{tabular}

Fuente: Microempresa Yoha's Style, enero 2020

En la tabla 6, se observa el estado de resultados y el correspondiente análisis vertical en relación con las ventas con un total de $\$ 16.560,82$; los costos de producción y ventas poseen un $75,67 \%$ de la inversión, mientras que el $24,33 \%$ de utilidad bruta en ventas para hacer frente a gastos tales como publicidad y propaganda, combustibles, lubricantes entre otros. La utilidad neta del ejercicio corresponde al 19,34\%; es decir, 3.203,17. Los resultados del costos de producción coinciden con lo que menciona Gavidia y Miranda (2018) que entre las principales debilidades del sector calzado se enfrenta a la producción en cantidades pequeñas, orientación de producción por altos costos, bajo nivel de tecnificación, entre otros.

Tabla 6

Estado de resultados (expresado en \$)

\begin{tabular}{llcc}
\hline \multicolumn{3}{c}{ MICROEMPRESA YOHA'S STYLE } & \\
\hline \multicolumn{3}{c}{ DEL 01 AL 31 DE ENERO DEL 2020 } & Análisis \\
& & de ventas \\
& & \\
4. & INGRESOS & \\
4.1. & Ventas de bienes & & \\
4.1 .01 .02 & Ventas Iva 12\% & & \\
5. & COSTOS Y GASTOS & & \\
5.1 & COSTOS & & \\
5.1 .01 & Costo de producción y ventas & $\$ 12.531,76$ & $75,67 \%$ \\
& Utilidad bruta en ventas & $\$ 4.029,06$ & $24,33 \%$ \\
5.2 & GASTOS & & \\
5.2 .02 .03 & GASTOS GENERALES & & \\
\hline
\end{tabular}


Tabla 6

Estado de resultados (expresado en \$) (continuación)

\begin{tabular}{llrr}
\hline \multicolumn{4}{c}{ MICROEMPRESA YOHA'S STYLE } \\
\hline \multirow{4}{*}{ DEL 01 AL 31 DE ENERO DEL 2020 } \\
5.2 .03 .07 & Publicidad y propaganda & $\$ 45,00$ & $0,27 \%$ \\
5.2 .03 .14 & Combustibles y lubricantes & $\$ 30,00$ & $0,18 \%$ \\
5.2 .03 .15 & Depreciación maquinaria & $\$ 185,63$ & \\
& Total gastos generales & $\$ 260,63$ & $1,57 \%$ \\
& Ganancia antes del 15\% & & \\
& trabadores e impuestos & $\$ 3.768,43$ & $22,76 \%$ \\
& 15\% trabajadores & $\$ 565,26$ & $3,41 \%$ \\
& Ganancia neta del ejercicio & $\$ 3.203,17$ & $19,34 \%$ \\
\hline
\end{tabular}

Fuente: Microempresa Yoha's Style, enero 2020

La tabla 7 muestra los indicadores de liquidez aplicados en la microempresa Yoha's Style. El índice de liquidez corriente indica que la empresa, por cada dólar de pasivos corrientes posee $\$ 17,7$ en sus activos corrientes para hacer frente a sus obligaciones a corto plazo, este resultado refleja que la microempresa mantiene un alto índice de liquidez en el ejercicio analizado; además, la capacidad de pago a corto plazo es inmediata; es decir, existe una alta solidez financiera. Para González et al. (2008) al existir solidez financiera, las posibilidades de cancelar a los proveedores o acreedores son altas, lo cual es importante poseer los recursos financieros suficientes para conseguir los servicios o materias primas necesarias para la elaboración del producto final y garantizar la supervivencia del negocio. Al aplicar el indicador de prueba ácida; es decir, ser más exigentes, al restar los inventarios, la empresa mantiene el $\$ 6,3$ por cada dólar de deuda a corto plazo; es decir, sigue siendo liquida. Es importante la liquidez, ya que con ella se garantiza el retorno de la inversión a los propietarios o inversionistas empresariales.

Tabla 7

Indicadores de Liquidez

\begin{tabular}{lcc}
\hline Indicadores & \multicolumn{2}{c}{ Resultados } \\
\hline 1. Liquidez corriente: & $\underline{71.394,98}$ & 17,7 \\
& $4.044,12$ & \\
2. Prueba ácida: & $\underline{25.584,70}$ & 6,3 \\
& $4.044,12$ & \\
\hline
\end{tabular}

Fuente: Microempresa Yoha's Style, enero 2020

De otra parte, en la tabla 8 , los indicadores de solvencia, se evidencia la independencia que posee la empresa con respecto a sus acreedores ya que sólo el $18 \%$ de sus activos están comprometidos con los acreedores y el 22\% del patrimonio; decir, el mayor financiamiento corresponde de los propietarios de la empresa. Por cada unidad invertida en el patrimonio la microempresa posee \$2,48 en sus activos fijos netos. En el análisis 
del apalancamiento y apalancamiento financiero no difieren el uno del otro ya que no existe intereses financieros. En este sentido la empresa por cada $\$ 1,22$ que posee en activos fueron financiados por los recursos internos de la microempresa y en menor proporción por terceros.

Tabla 8

Indicadores de Solvencia

\begin{tabular}{|c|c|c|}
\hline Indicadores & \multicolumn{2}{|c|}{ Resultados } \\
\hline \multirow[t]{2}{*}{ 1. Endeudamiento del Activo: } & $19.044,12$ & \multirow{2}{*}{0,18} \\
\hline & $106.739,35$ & \\
\hline \multirow[t]{2}{*}{ 2. Endeudamiento Patrimonial: } & $19.044,12$ & \multirow{2}{*}{0,22} \\
\hline & $87.695,23$ & \\
\hline \multirow[t]{2}{*}{ 3. Endeudamiento del Activo Fijo: } & $87.695,23$ & \multirow{2}{*}{2,48} \\
\hline & $35.344,37$ & \\
\hline \multirow[t]{2}{*}{ 4. Apalancamiento: } & $106.739,35$ & \multirow{2}{*}{1,22} \\
\hline & $87.695,23$ & \\
\hline \multirow[t]{2}{*}{ 5. Apalancamiento Financiero: } & 0,04 & \multirow{2}{*}{1,22} \\
\hline & 0,03 & \\
\hline
\end{tabular}

Fuente: Microempresa Yoha's Style, enero 2020

En otra instancia en la tabla 9 en los indicadores de rentabilidad, se obtiene como resultado que la microempresa genera $3 \%$ en utilidades por cada unidad monetaria invertido en activos. Por otra parte, el margen bruto que genera la empresa es del $24 \%$ para cubrir gastos operativos y otras deducciones adicionales. En cuanto al margen operacional la microempresa por cada dólar en ventas realizadas genera un valor del \$0,23 centavos de dólares por utilidad antes del $15 \%$ a trabajadores e impuestos. En concordancia con Artieda (2015) los resultados económicos en los estados financieros muestran a las empresas el camino hacia el desarrollo y crecimiento empresarial. Asimismo, en el indicador de rentabilidad neta de ventas la microempresa genera $19 \%$ de utilidad neta en relación con las ventas realizadas. El indicador de rentabilidad operacional del patrimonio establece que los propietarios obtienen un $4 \%$ de utilidad sin considerar la deducción de participación a trabajadores con relación al capital invertido, porcentaje que coincide con la rentabilidad financiera convirtiéndose en el beneficio neto que recibe el propietario de la microempresa. 
Tabla 9

Indicadores de Rentabilidad

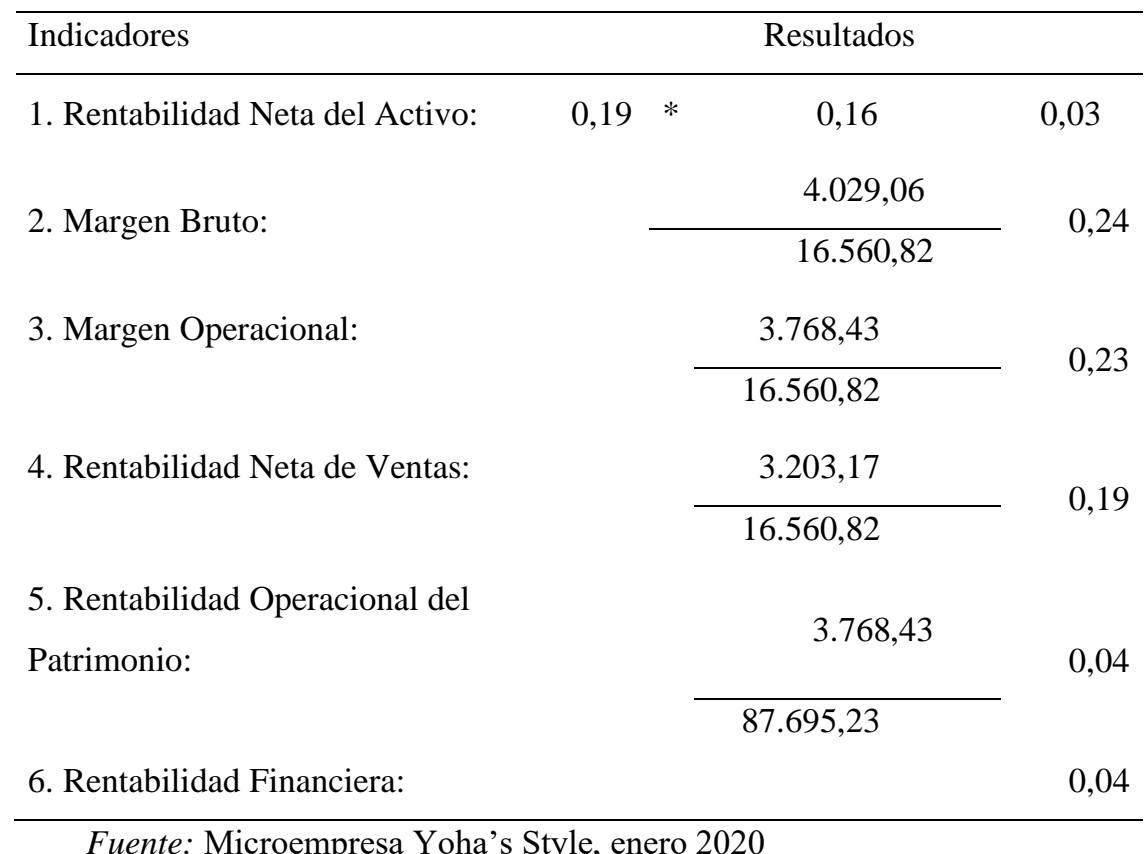

Fuente: Microempresa Yoha's Style, enero 2020

\section{Conclusiones}

- Del estudio realizado se deriva que el rendimiento operacional de la microempresa en relación con el patrimonio es muy bajo al obtener tan sólo el $4 \%$ como retorno a los propietarios dada la inversión realizada; la misma situación se observa en la rentabilidad neta del activo y rentabilidad financiera que generan tan sólo el 3\% y $4 \%$ respectivamente. Dicha información refleja que la administración debe establecer estrategias que brinden mayor productividad al menor costo posible. La contabilidad de costos es una herramienta en la gestión de las empresas sin considerar su tamaño, en especial en los procesos de producción; pero, al estar categorizadas con el término tributario como no obligada a llevar contabilidad; desconoce de los beneficios y los efectos que ocasiona en la administración.

- La microempresa debe implementar la contabilidad de costos ya que proporciona información cuantitativa que sirve para medir los resultados sobre cómo se administran los recursos de materia prima, insumos y otros materiales, así como los costos de mano de obra, costos indirectos de fabricación y los gastos de operación.

- Es indudable que las microempresas son generadoras de empleo; pero, al no aplicar contabilidad limita su crecimiento, desarrollo y toma decisiones oportunas en mercados competitivos y globalizados.

\section{Referencias Bibliográficas}

Artieda, C. (2015). Análisis de los sistemas de costos como herramientas estratégicas de gestión en las pequeñas y medianas empresas (PYMES). Revista Publicando, 2(3), 
90-113.

Baena, D. (2014). Análisis financiero, Enfoque y proyecciones. https://books.google.com.ec/books?id=1Xs5DwAAQBAJ\&pg=PA30\&dq=estados +financieros+son+una+representación+financiera\&hl=es\&sa=X\&ved=2ahUKEwi o3PzVpfTtAhWQo1kKHZDsBn0Q6AEwAHoECAQQAg\#v=onepage\&q=estados financieros son una representación fi\&f=false

Barreto, N. B. (2020). Análisis Financiero: factor sustancial para la toma de decisiones en una empresa del sector comercial. Universidad y Sociedad, 12(3), 129-134. https://doi.org/https://orcid.org/0000-0001-7232-7131

Correa, F., Leiva, V., \& Stumpo, G. (2018). Mipymes y heterogeneidad estructural en América Latina. Comisión Económica para América Latina y el Caribe (CEPAL), October, https://www.researchgate.net/publication/328314810_Mipymes_y_heterogeneidad _estructural_en_America_Latina

Escobar, F., Argota, G., Ayaviri, V. D., Aguilar, S. L., Quispe, G. M., \& Arellano, O. E. (2021). Costeo basado en actividades (ABC) en las PYMES e iniciativas innovadoras: ¿opción posible o caduca? Revista de Investigaciones Altoandinas Journal of High Andean Research, 23(3), 171-180. https://doi.org/https://doi.org/10.18271/ria.2021.321

García, Y. M. (2021). Las MiPymes como factor determinante del emprendimiento: un análisis bibliométrico para el periodo 2000- 2020. INNOVA Research Journal, 6(3), 79-93. https://doi.org/https://doi.org/10.33890/innova.v6.n3.1.2021.1828

Gavidia, H. P., \& Miranda, J. C. (2018). costs of the MyPES of the footwear of Porvenir - Trujillo - 2016. Económicas, Facultad De Ciencias Trujillo, Universidad Nacional De Juan, Av Ii, Pablo Universitaria, Ciudad, 14(1), 53-63. file://C:/Users//Downloads/1920-Texto del artículo-5617-1-10-20180705.pdf

Genoni, G., \& Zurita, S. (2004). Capital de trabajo, gestion de tesoreria y valuacion de empresas. 11 39-54. http://repositorio.uchile.cl/bitstream/handle/2250/127322/E_A_2004_Vol11_N1_ Genoni.pdf? sequence $=1 \&$ isAllowed $=\mathrm{y}$

González, P. G., Universidad, E., \& Industrial, I. (2008). Una aproximación al modelo de toma de decisiones usado por los gerentes de las micro, pequeñas y medianas empresas ubicadas en Cali , Colombia desde un enfoque de modelos de decisión e indicadores financieros y no financieros *. Contaduría Universidad de Antioquia, $52,131-154$.

Gutiérrez, C., \& Gutiérrez, E. (2017). Obligación tributaria e incidencia en la conformación de microempresas en el sur de la ciudad de Guayaquil. En Espirales Revista Multidisciplinaria de investigación (Vol. 1, Número 8). 
https://www.revistaespirales.com/index.php/es/article/view/78

Henández, R., Fernández, C., \& Baptista, M. (2014). Metodología de la Investigación.

Hurtado, K. del R. (2020). Sistemas de costo, logística inversa y gestión sostenible en empresasSistemas de costo, logística inversa industriales. Cooperativismo $y$ Desarrollo, 8(3), 526-537. http://coodes.upr.edu.cu/index.php/coodes/article/view/354\%0Ahttp://files/250/Ga rcía - 2020 - Sistemas de costo, logística inversa y gestión sos.pdf

Instituto Nacional de Estadisticas y Censos. (2021). Visualizador de Estadísticas Empresariales - Instituto Nacional de Estadística y Censos (INEC) | Tableau Public. https://public.tableau.com/profile/instituto.nacional.de.estad.stica.y.censos.inec.\#!/ vizhome/VisualizadordeEstadisticasEmpresariales/Dportada

Kato Maldonado, L. (2013). La problemática de la ganancia en la economía y su relación con la contabilidad empresarial. Economía Informa, 383, 23-63. https://doi.org/10.1016/s0185-0849(13)71340-9

Laveriano, W. (2010). Importancia del control de inventarios en la empresa Ficha Técnica. 2010. http://biblioteca.esucomex.cl/RCA/Importancia del control de inventarios en la empresa.pdf

Medina, S. A., Silvia, A., Ruata, A., Segundo, A., Contreras, F., Benita, P., Carmen, D., De Aguirre, C., \& De Costos, C. (2018). Contabilidad de Costos. En cidepro.org. www.cidepro.org

Mejía, C., \& Higuita, C. (2015). Cost to serve as a strategic decision variable in the design of strategies as regards emerging marketing channels. Estudios Gerenciales, 31(134), 50-61. https://doi.org/http://dx.doi.org/10.1016/j.estger.2014.08.006

Morales, L., Morales, K., \& Valle, A. (2017). Crecimiento y supervivencia empresarial en el sector de fabricación de calzado en Ecuador. Panorama Económico, 25(1), 4356. https://doi.org/10.32997/2463-0470-vol.25-num.1-2017-2068

Muñoz Briones, J. C. (2018). Análisis a los estados financieros de empresas camaroneras en la provincia de El Oro, Ecuador. INNOVA Research Journal, 3(9), 112-125. https://doi.org/10.33890/innova.v3.n9.2018.686

Nava, R., \& Marbelis, A. (2009). Análisis financiero : una herramienta clave para una gestión financiera eficiente. Venezolana de Gerencia (RVG), 606-628. https://www.redalyc.org/pdf/290/29012059009.pdf

Palacios, A., \& Barreto, A. (2014). NIC 16: Desglosando la Propiedad, Planta y Equipo. Apuntes contables, 17, 37-57. https://core.ac.uk/download/pdf/230105103.pdf

Porporato, M. (2015). Contabilidad de gestión para controlar o coordinar en entornos turbulentos: Su impacto en el desempeño organizacional. Contaduria y 
Administracion, $60(3)$

511-534. https://doi.org/https://doi.org/10.1016/j.cya.2015.02.002 .

Quezada, V., Seck, J. C., Quezada, J. C., \& Cuatepotzo, A. (2020). Sistema de producción multi-línea optimizado por PSO. Ingeniería Investigación y Tecnología, 21(1), 1-11. https://doi.org/https://doi.org/10.22201/fi.25940732e.2020.21n1.006

Santana, L. (2017). Determinantes de la supervivencia de microempresas en Bogotá: un análisis con modelos de duración. scielo.org.co, 27. https://doi.org/10.15446/innovar.v27n64.62368

Superintendencia de Compañías del Ecuador. (2021). Tabla de Indicadores. En https://www.supercias.gob.ec/bd_supercias/descargas/ss/20111028102451.pdf. https://doi.org/10.2307/j.ctv1dp0wtc.10

Texis, M., Ramírez, M., \& Aguilar, J. (2016). Microempresas de base social y sus posibilidades de supervivencia. Contaduria y Administracion, 61(3), 551-567. https://doi.org/http://dx.doi.org/10.1016/j.cya.2015.04.001

Vega, S., \& Tello, J. (2013). El discurso directivo en la comunicación de los estados financieros. El caso de dos empresas chilenas. 59(3), 261-283. https://doi.org/http://dx.doi.org/10.1016/S0186-1042(14)71272-0

Villarreal, F., \& Rincón, C. A. (2009). Método Matricial FHER en un Sistema de Costos por Procesos, o en Línea. Entramado, 5(2), 106-132. https://www.redalyc.org/articulo.oa?id=265419724008

Wild, J. J., Subramanyam, K. R., \& Halsey, R. (2007). Análisis de estados financieros. En Mc Graw Hill. http://fullseguridad.net/wp-content/uploads/2016/11/Análisis-deestados-financieros-9na-Edición-John-J.-Wild.pdf

Yasuní, R. (2016). Plan Nacional de Desarrollo 2017-2021-Toda una Vida. www.planificacion.gob.ec

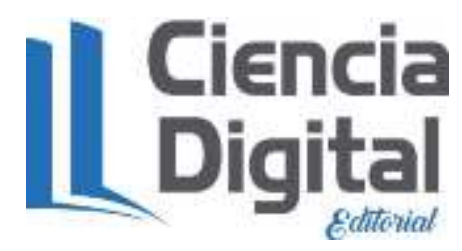




\section{PARA CITAR EL ARTÍCULO INDEXADO.}

Delgado Olaya, R. M., Yánez Sarmiento, M. M., Orellana Arévalo, B. L., Camacho Yunga, J. M., \& Espinoza Criollo, K. E. (2021). La contabilidad de costos como herramienta de gestión en la microempresa Yoha's Style. ConcienciaDigital, 4(4.2), 6076. https://doi.org/10.33262/concienciadigital.v4i4.2.1940

\section{ULiencia}

El artículo que se publica es de exclusiva responsabilidad de los autores y no necesariamente reflejan el pensamiento de la Revista Conciencia Digital.

El artículo queda en propiedad de la revista y, por tanto, su publicación parcial y/o total en otro medio tiene que ser autorizado por el director de la Revista Conciencia Digital.

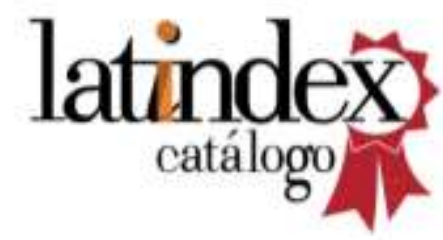

